\title{
PROCEDIMIENTO DE ESCISIÓN ELECTRO-QUIRÚRGICA POR ASA TÉRMICA Y RIESGO DE PARTO PREMATURO. UNA REVISIÓN SISTEMÁTICA DE LA LITERATURA
}

\author{
Milenko Pavlovic B. ${ }^{a}$, Francisco Arancibia M.a , Eduardo Mordojovich Z.. , José Antonio \\ González G. ${ }^{1}$, Luis Villavicencio F. ${ }^{1}$ \\ 1 Unidad de Patología Cervical, Departamento de Ginecología y Obstetricia, Hospital Parroquial de San Bernardo.
}

a Alumnos, Escuela de Medicina, Universidad de los Andes.

\section{RESUMEN}

Antecedentes: A diferencia de la conización cervical por cono frío, no se ha podido demostrar una clara asociación entre el procedimiento de escisión electro-quirúrgica por asa térmica (LEEP) y el riesgo de parto prematuro. Objetivo: Análisis crítico de la literatura científica, en relación al riesgo de presentar un parto prematuro en pacientes que han sido sometidas a LEEP, y los resultados materno-perinatales asociados. Búsqueda sistemática en múltiples bases de datos. Resultados: Se encontraron sólo tres artículos que cumplían los criterios de inclusión, los cuales son incluidos en esta revisión. De éstos, el primero muestra que el LEEP no aumenta el riesgo de parto prematuro ni de recién nacidos de bajo peso. El segundo evidencia un aumento del riesgo de rotura prematura de membranas y parto prematuro secundario a esto, pero no de parto prematuro espontáneo. Sin embargo, el tercero, señala que el LEEP se asocia en forma significativa a riesgo aumentado de parto prematuro, parto prematuro secundario a rotura prematura de membranas y recién nacidos de bajo peso. Los tres estudios son de cohortes retrospectivas, lo cual les otorga un nivel de evidencia de tipo $2 \mathrm{~b}$. Conclusión: La evidencia indica que la excisión de la zona de transformación a través del uso de LEEP está asociada a un pequeño, pero real incremento del riesgo de presentar un parto de prétermino.

\section{PALABRAS CLAVE: Neoplasia intra-epitelial cervical, conización cervical, procedimiento de escisión electroquirúrgica por asa térmica, parto prematuro, rotura prematura de membranas}

\section{SUMMARY}

Background: Unlike cold-knife conization, studies have shown conflicting results on the outcome of pregnancy following loop electrosurgical excision procedure (LEEP). Objective: Critical analysis of the literature to establish if the LEEP treatments increase risk of preterm delivery and its influence in maternal-perinatal results. Results: Only three studies were filling the inclusion criteria. The first study does not show that LEEP treatment increase risk of preterm delivery and low birth weight. The second study shows increased risk of premature rupture of membranes and the subsequent preterm delivery, but not spontaneous preterm delivery. Nevertheless the third study shows increased risk of premature rupture of membranes and the subsequent preterm delivery, spontaneous preterm delivery and low birth weight. The three studies are based in retrospective cohorts, which grant them a level of evidence of type $2 b$. 
Conclusion: The evidence indicates that loop excision of the transformation zone by LEEP is associated with a small but real increase the risk of preterm delivery.

\section{KEY WORDS: Cervical intra-epithelial neoplasia, cervical conization, loop electrosurgical excision procedure, preterm delivery, premature rupture of membranes}

\section{INTRODUCCIÓN}

La neoplasia intraepitelial (NIE) del cuello uterino, es un estado precanceroso que sin tratamiento oportuno evoluciona a cáncer cervicouterino. Constituye una patología de gran importancia en salud pública, debido a que es la cuarta causa de mortalidad global por cáncer en las mujeres chilenas y la más frecuente en mujeres entre 35 y 54 años. La tasa de mortalidad por éste cáncer fue de 8,4 por 100 mil mujeres en 2003, con un total de 677 muertes. El tamizaje mediante la citología de Papanicolaou (PAP) y el oportuno tratamiento de la lesión preinvasora, permite una curación con un alto grado de efectividad, constituyéndose en la intervención de mayor costo efectividad para prevenir el desarrollo de cáncer cervicouterino invasor (1). Las tasas de incidencia según edad para carcinoma cervical in situ son mayores para mujeres entre los 15 a 44 años de edad, lo que coincide con el periodo reproductivo de éstas, con un alza de 200 casos por 100 mil mujeres a los 25 años de edad (2).

El parto prematuro ha sido definido por la OMS como el que ocurre antes de las 37 semanas de gestación, siendo su límite inferior de edad gestacional las 22 semanas y/o $500 \mathrm{~g}$ de peso fetal. Es la causa más importante de morbilidad y mortalidad perinatal, siendo su incidencia del 8 al $10 \%$ de los partos (3).

El objetivo de los tratamientos hoy disponibles para NIE es remover las células anormales de la zona de transición cervical y, al mismo tiempo, conservar la función cervical. Para esto existen diversas técnicas como el cono frío, la ablación láser, la conización láser y el procedimiento de escisión electro-quirúrgica por asa térmica (LEEP).

EI LEEP fue descrito como tratamiento de NIE por Prendiville y cols (4) en 1989. Esta técnica es ampliamente utilizada en el mundo, remueve menos tejido, requiere menor tiempo operatorio y esta asociada a menores tasas de hemorragia, dolor e infección, que las otras técnicas convencionales para tratamiento de NIE, demostrando igual efectividad $(5,6)$.

Se ha visto en numerosos estudios que el tratamiento de la NIE con cono frío aumentaría el riesgo de partos prematuros y bajo peso al nacer, no existiendo aún una clara asociación entre éstos factores y el $\operatorname{LEEP}(7,8,9)$.

Debido a la alta prevalencia de NIE en las mujeres en edad fértil junto con la trascendental utilidad del cono en el tratamiento de ésta, y al gran problema que implica el parto prematuro en la morbimortalidad perinatal, nos planteamos la necesidad de aclarar la asociación existente entre LEEP y parto de pretérmino.

\section{MATERIAL Y MÉTODO}

Se planteó la siguiente interrogante: ¿Es el LEEP un factor de riesgo para parto prematuro? Para responder a ésta, se realizó una revisión de las bases de datos Pubmed, Ebsco, Cochrane Library y EMBASE, utilizando las siguientes palabras claves: "obstetric labor, premature" AND "conization", "loop electrosurgical excision" AND "obstetric labor, premature", "loop electrosurgical excision" AND "complication", utilizándose además el término "prognosis". Se incluyeron los estudios que cumpliesen con los criterios de inclusión propuestos para ésta revisión (Tabla I).

\section{RESULTADOS}

Sólo 3 estudios fueron incluidos según los criterios propuestos (Tabla II), los cuales se describen a continuación.

1. Pregnancy outcome after loop electrosurgical excision procedure for the management of cervical intraepithelial neoplasia (10). Estudio de cohorte retrospectivo de mujeres que se han realizado un LEEP como confirmación biópsica para NIE entre diciembre de 1995 y diciembre de 2000 y que posteriormente tuvieron un embarazo mayor a 20 semanas de gestación en la University Hospital of Northern Norway. Se incluyeron sólo mujeres menores de 45 años, obteniéndose de ésta forma 79 pacientes sometidas a LEEP y 158 tomadas como grupo control. Método: Se realizó emparejamiento de dos controles con un caso para la fecha de parto, edad, paridad, historia obstétrica previa y el hábito de fumar. Las variables primarias estudiadas 


\section{Tabla I}

\section{CRITERIOS DE INCLUSIÓN PARA TRABAJOS QUE RELACIONAN LEEP CON PARTO PREMATURO}

\begin{tabular}{l} 
Criterios utilizados \\
\hline - Estudios de cohorte con pérdidas menores al $20 \%$ de \\
pacientes \\
- Control de variables confundentes \\
- Sujetos a estudiar: \\
Mujeres sometidas a LEEP cursando su primer emba- \\
razo post procedimiento, mayor a 20 semanas de ges- \\
tación, sin factores de riesgo mayores para parto pre- \\
maturo \\
- Sujetos a comparar: \\
Mujeres sin antecedentes de NIE o de procedimiento \\
para tratamiento de ésta, cursando un embarazo ma- \\
yor a 20 semanas sin factores de riesgo mayores para \\
parto prematuro \\
- Outcome principal: Incidencia de partos prematuros \\
\hline
\end{tabular}

Tabla II

\section{TRABAJOS QUE RELACIONAN LEEP CON PARTO PREMATURO Y CUMPLEN CON LOS CRITERIOS DE INCLUSIÓN}

\author{
Trabajos incluidos en ésta revisión
}

(10) Acharya G, Kjeldberg I, Hansen SM, Sørheim N, Jacobsen BK, Maltau JM. Pregnancy outcome after loop electrosurgical excision procedure for the management of cervical intrapithelial neoplasia. Arch Gynecol Obstet 2005;272(2):109-12.

(11) Sadler L, Saftlas A, Wang W, Exeter M, Whittaker J, McCowan L. Treatment for Cervical Intraepithelial Neoplasia and Risk of Preterm Delivery. JAMA 2004;291(17): 2100-6.

(12) Samson SL, Bentley JR, Fahey TJ, McKay DJ, Gill $\mathrm{GH}$. The effect of loop electrosurgica I excision procedure on future pregnancy outcome. Obstet Gynecol 2005;105(2):325-32.

son la duración del embarazo y el peso al nacer. Otras variables revisadas fueron el grado de displasia cervical, tamaño del LEEP, complicaciones del embarazo y tipo de parto. La edad gestacional fue determinada por ultrasonografía midiendo el diámetro biparietal antes de las 20 semanas. Un parto antes de las 37 semanas fue definido como prematuro y el peso al nacer menor a $2.500 \mathrm{~g}$ como bajo peso. Para las variables continuas la diferencia entre el caso y el promedio entre los dos controles fue realizada por t-test para comparaciones en pares. Se comprobó correlaciones con la correlación producto momento de Pearson. Se utilizó un valor $\mathrm{p}<0,05$ para significancia estadística y se estimó el Odds Ratio (OR) con 95\% de intervalo de confianza (Cl). Resultados: La edad promedio al momento del LEEP fue de 27 años. No se observaron diferencias significativas entre los grupos en lo que respecta a la tasa de partos prematuros y al peso de nacimiento. Hubo diferencias significativas en las complicaciones relacionadas con el embarazo, siendo mayores en el grupo LEEP ( $20 \%$ vs. $7 \%$; $p=0,006$ ). Los tipos de complicaciones evaluadas fueron infecciones, incompetencia cervical, contracciones prematuras, pre-eclampsia y diabetes gestacional. Se vio una relación inversa entre el tamaño del LEEP y la duración del embarazo, diferencia no significativa. Con un tamaño de LEEP menor a $15 \mathrm{~mm}$, la media de la edad gestacional fue 0,6 semanas mayor en casos que en los controles. Con LEEP mayor a $25 \mathrm{~mm}$, la edad gestacional fue 2 semanas menor en casos que en controles. Una relación similar se vio con el peso al nacer, pero sin significancia estadística. El riesgo de parto prematuro (OR 4; 95\% Cl 1,0-16,0; $\mathrm{p}=0,05$ ) y el bajo peso al nacer (OR 14; $95 \% \mathrm{Cl}$ $1,7-114 ; p=0,01$ ) fueron significativamente mayores cuando el tamaño del LEEP fue mayor a $25 \mathrm{~mm}$ comparado con controles, no dándose esta relación en forma significativa para el total de LEEP.

Validación del estudio: Presenta una muestra homogénea en donde se emparejaron las pacientes según características similares, bien definida y representativa de la población en estudio. Los factores confundentes fueron eliminados. Presenta un periodo de recolección de datos de 5 años, siendo éstos bien definidos, objetivos y obtenidos todos de un mismo centro. Presenta pocas tablas con resúmenes de los resultados y muchos de ellos no son claros. Se puede criticar que la muestra es pequeña y el estudio se realizó sólo en un centro clínico, por lo que éstos resultados son difíciles de extrapolar a otras poblaciones. Se trataría de un estudio de pronóstico de cohorte retrospectiva según el centro de medicina basada en la evidencia de Oxford, con un nivel de evidencia y fuerza de recomendación 2b B (13).

2. Treatment for cervical intraepithelial neoplasia and risk of preterm delivery (11). Estudio de cohorte retrospectivo, realizado en el National Women's Hospital, Auckland, New Zealand, entre los años 1988 y 2000. Se incluyeron en el estudio todas aquellas mujeres vistas o tratadas en la clínica de colposcopia 
antes de su fecha de última menstruación, que posteriormente cursaron con un embarazo mayor a 20 semanas de gestación y parto controlado en el hospital. Criterio de exclusión fueron mujeres tratadas con otros métodos que no fuesen ni LEEP ni conización láser, pacientes tratadas antes de 1988 y pacientes con tratamiento poco claro. Se incluyeron en este estudio 426 pacientes para grupo control y 652 mujeres tratadas, de éstas últimas, 278 pacientes fueron tratadas con LEEP. Método: La variable primaria a estudiar fueron los partos prematuros y sus subtipos: parto prematuro espontáneo, rotura prematura de membranas que lleva a parto prematuro y parto prematuro iatrogénico. Para identificar los posibles factores confundentes, que modifican la asociación entre la aplicación del LEEP y el parto prematuro, se utilizó el análisis de regresión lineal. Para identificar el riesgo de parto prematuro se aplicó el análisis multivarianza de riesgo relativo $(R R)$, con un valor $p<0,05$ para la significación estadística. Resultados: La tasa global de parto de pretérmino fue de $13,8 \%(n=149)$, de estos $41(27,1 \%)$ fueron esponténeos, $41(27,5 \%)$ iatrogénicos y $67(45 \%)$ secundarios a rotura prematura de membranas. Las tasas globales de parto prematuro de pacientes tratadas exceden a las no tratadas, sin tener significancia estadística (14,9\% vs. $12,2 \%$; $p=0,21)$. Lo mismo ocurre en los subgrupos de parto prematuro espontáneo $(4,0 \%$ vs. $3,5 \% ; p=0,64)$ y iatrogénico (2,9\% vs. $5,2 \% ; p=0,09)$. En el caso del parto prematuro secundario a rotura prematura de membranas, se observa una tasa mayor de parto de pretérmino en las pacientes tratadas (8,4\% vs. $3,5 \%$; $p=0,004)$, la cual es estadísticamente significativa. El análisis de RR ajustado muestra que el tratamiento de NIE con LEEP, no se asocia a un riesgo aumentado en forma global de parto prematuro (RR 1,2; 95\% $\mathrm{Cl} 0,8-1,8)$ ni a parto prematuro espontáneo (RR 1,5; $95 \% \mathrm{Cl} 0,7-3,2)$. Cuando se analiza el RR de parto prematuro secundario a rotura prematura de membranas en pacientes con NIE tratadas mediante LEEP, se aprecia un riesgo significativamente aumentado (RR 1,9; 95\% Cl 1,0-3,8).

Validación del estudio: Presenta una muestra homogénea en donde se emparejaron las pacientes según características similares. La muestra esta bien definida, con criterios de inclusión estrictos y es representativa de la población en estudio. Los factores confundentes fueron eliminados, el tiempo de recolección de datos fue de 12 años, obteniéndose todos de un mismo centro, utilizándose una base de datos completa y confiable. Presenta tablas con resúmenes de los resultados, cada variable con su significancia estadística. Se puede criticar que el estudio se realizó sólo en un centro y utilizó criterios de inclusión estrictos, lo que le resta validez externa, así lo resul- tados pueden ser extrapolables a la población en estudio, pero es difícil extrapolarlos a otras. Se trataría de un estudio de pronóstico de cohorte retrospectiva según el centro de medicina basada en la evidencia de Oxford, con un nivel de evidencia y fuerza de recomendación $2 \mathrm{~b} B$ (13).

3. The effect of loop electrosurgical excision procedure on future pregnancy outcome (12). Estudio de cohorte retrospectivo realizado en el Departments of Obstetrics and Gynecology, Dalhousie University, Halifax, Nova Scotia, y el Dr. Everett Chalmers Hospital, Fredericton, New Brunswick. Las cohortes se obtuvieron de 2 bases de datos, la primera es la Provincial Cytology/Colposcopy Registry que contiene información de todas las mujeres que se han realizado una colposcopia y tratamiento de NIE en Nova Scotia desde 1992. La segunda base de datos es la Nova Scotia Atlee Perinatal Database, que contiene información de todos los embarazos mayores a 20 semanas de gestación con sus respectivos partos e información perinatal en Nova Scotia desde 1988. Se recolectaron datos de la primera base desde 1992 hasta 1999, extrapolándolos con los de la segunda base de datos. El grupo LEEP ( $n=571)$ fueron todas las mujeres sometidas a LEEP que cursaron un embarazo mayor a 20 semanas luego del procedimiento, incluyéndose sólo los datos del primer embarazo pos LEEP. El grupo de comparación $(n=571)$ fueron mujeres sin historia de cirugía cervical que cursaron un embarazo mayor a 20 semanas. Método: La variable primaria a estudiar fue el parto de pretérmino, que se subdividió en 2 grupos, menores de 34 y menores de 37 semanas. Otras variables estudiadas fueron recién nacido de bajo peso $(<2.500 \mathrm{~g})$, rotura prematura de membranas de pretérmino, profundidad y diámetro del LEEP. Las pacientes fueron emparejadas por edad, paridad, fecha de parto y consumo de tabaco. Se excluyeron de ambos grupos mujeres con factores de riesgo mayores para parto prematuro como el antecedente de éste y embarazos múltiples. Para comparar diferencias en variables dicotómicas se utilizó el test de McNemar, para las variables continuas se usó t-test o la prueba de Wilcoxon. Se utilizó un valor $p<0,05$ para significancia estadística y se estimó el Odds Ratio (OR) con 95\% de intervalo de confianza (Cl). Resultados: Para la variable parto prematuro, existió una diferencia estadísticamente significativa entre los grupos LEEP y control (7,9\% vs $2,5 \%$; $p=0,001)$. El riesgo de presentar un parto de pretérmino en el grupo LEEP fue también significativo (OR 3,5; 95\% Cl 1,9-6,95). Para la variable parto prematuro menor a 34 semanas, no hubo diferencia significativa entre los grupos estudiados $(1,25 \%$ vs. $0,36 \% ; p=0,12)$ y tampoco existió un riesgo significativamente aumentado (OR 3,5; 95\% Cl 0,85-23,5). Se evidenció una 
diferencia significativa entre el grupo LEEP y control en lo que refiere a $\mathrm{RN}$ de bajo peso $(5,4 \%$ vs. $1,9 \%$; $\mathrm{p}=0,003$ ), cuyo riesgo también fue significativo (OR 3; $95 \% \mathrm{Cl} 1,52-6,46)$. También se vio que las pacientes LEEP presentaron en forma significativa una mayor cantidad de partos prematuros secundarios a rotura prematura de membranas $(3,5 \%$ vs $0,9 \% ; p=0,004)$, con un riesgo igualmente significativo (OR 4,$1 ; 95 \%$ $\mathrm{Cl} 1,48-14,09)$. No se apreciaron diferencias significativas entre el diámetro $(18,2$ vs. 20,$2 ; p=0,48)$, la profundidad $(6,5$ vs. 6,$8 ; p=0,65)$ y el intervalo entre el LEEP y el parto (1752 vs. $1113 ; p=0,3$ ) en las mujeres sometidas a LEEP que resultaron con parto pretérmino y las que no.

Validación del estudio: Presenta una muestra homogénea, con cohortes bien definidas y representativas del universo. Los factores confundentes fueron eliminados, el tiempo de recolección de datos fue de 7 años y se utilizaron bases de datos confiables de dos centros distintos. Presenta tablas con resúmenes de los resultados, cada variable con su significancia estadística. El emparejamiento entre el grupo LEEP y el de comparación fue adecuado, el $n$ del trabajo (571 por grupo) da un poder estadístico suficiente para poder validar los resultados. Las limitantes de este estudio son las propias que existen para cualquier estudio de cohorte retrospectivo bien realizado. Se trataría de un estudio de pronóstico de cohorte retrospectiva según el centro de medicina basada en la evidencia de Oxford, con un nivel de evidencia y fuerza de recomendación $2 b \mathrm{~B}$ (13).

\section{DISCUSIÓN}

En esta revisión incluimos 3 estudios de cohorte retrospectivos con nivel de evidencia $2 b \mathrm{~B}$ cada uno de ellos. Acharya y cols (10), propone que el LEEP no aumentaría el riesgo de parto prematuro, a no ser que en este procedimiento se extraiga más de $25 \mathrm{~mm}$ de tejido cervical; también muestra que las complicaciones del embarazo aumentan pos LEEP. Samson y cols (12), muestra una clara evidencia de que el LEEP aumenta la incidencia de parto prematuro y de otras complicaciones perinatales, siendo su poder estadístico suficiente como para validar esta información, a diferencia del estudio de Acharya y cols (10), cuyo $\mathrm{n}$ es muy bajo y no tendría un poder estadístico suficiente para validar sus resultados. Además en el estudio de Samson y cols (12), se hizo una comparación de las pacientes tratadas con LEEP, entre las que presentaron parto de pretérmino y las que no, lo que demostró que no existía diferencia significativa entre los dos grupos en lo que respecta a la profundidad y diámetro del cono, por lo que los resultados expuestos por este grupo no estarían influenciados por la cantidad de tejido cervical extraído que, como fue propuesto por Acharya y cols (10), incidiría en el resultado perinatal. Sadler y cols (11), complementan los resultados obtenidos en el estudio de Samson, puesto que demuestra un aumento significativo de los partos prematuros asociados a rotura prematura de membranas, a pesar de que el parto de pretérmino como factor independiente no mostró asociación (11). Samson y cols (12), exponen que el riesgo aumentado de parto prematuro evidenciado en su análisis, se traduciría en un número necesario a tratar de 19 , es decir, de cada 19 pacientes sometidas a LEEP, 1 va a tener un parto prematuro. Otro dato que obtenemos de este estudio, es que el riesgo aumentado de parto prematuro tras un LEEP es significativo para partos menores a 37 semanas de edad gestacional, pero para los menores de 34 semanas, no se logró demostrar esta asociación. Esto es importante porque justamente éste grupo es el que está más expuesto a complicaciones. Samson y cols (12), también nos muestra un riesgo significativo de bajo peso al nacer para $\mathrm{RN}$ de madres que se sometieron a LEEP, lo que es importante ya que éstos tienen una morbimortalidad neonatal aumentada en comparación con los con peso normal.

Los tres estudios analizados fueron de cohortes retrospectivas. El diseño de estudio ideal para evaluar al LEEP como factor pronóstico en parto prematuro, sería un estudio de cohorte prospectiva, ya que éste permitiría seguir a las pacientes desde un inicio y analizar la relación entre LEEP y parto prematuro. Todos los estudios retrospectivos tienen limitaciones inherentes a éste tipo de diseño. Además, el hecho de que en estos tres estudios no se randomizara a las pacientes, hace posible a que los grupos comparados puedan diferir entre ellos, en otros aspectos distintos al antecedente de presentar una NIE y someterse a un LEEP. El hecho de que la información de las pacientes a estudiar derivara de bases de datos, predispone a ciertas limitaciones. Puede haber pérdida de datos, errores al ingresar y revisar los datos, y las limitaciones propias de las mismas bases de datos.

En conclusión podemos afirmar que los trabajos revisados nos permiten plantear la afirmación de que la excisión de la zona de transformación mediante el uso de LEEP como tratamiento para la NIE del cuello uterino, está asociada a un pequeño pero real incremento del riesgo de presentar un parto de prétermino $y$ de rotura prematura de membranas que termina en parto de pretérmino. La rotura prematura de membranas, es quizás la vía final común de los procesos patológicos que desencadenan un parto prematuro, sin embargo, el desconcierto que existe con respecto a la fisiopatología de este proceso dificulta en gran medida el poder realizar hipótesis acerca del mecanismo 
por el cual los procedimientos excisionales aumentan el riesgo de parto prematuro.

Finalmente, nuestras conclusiones se ven respaldadas por dos revisiones sistemáticas recientes de Arbyn y cols (8) y Kyrgiou y cols (9), que concluyen que todos los procedimientos excisionales, menos los procedimientos ablativos, se asocian a mayor riesgo de parto prematuro.

Esta información debería ser considerada por los médicos al momento de indicar un LEEP a mujeres en edad fértil con deseo futuro de fertilidad, especialmente a la hora de decidir el manejo de pacientes con NIE que deseen un embarazo a corto plazo y que tengan más de una alternativa terapéutica.

\section{BIBLIOGRAFÍA}

1. Ministerio de Salud. Guía Clínica Cáncer Cervicouterino. 1aㅡ ed. Santiago: MINSAL, 2005.

2. El-Bastawissi AY, Becker TM, Daling JR. Effect of cervical carcinoma in situ and its management on pregnancy outcome. Obstet Gynecol 1999;93(2):207-12.

3. Germain A, Carvajal J. Parto Prematuro. En: Pérez A, Donoso E (eds). OBSTETRICIA. $3^{\underline{a}}$ ed. Santiago: Editorial Mediterráneo, 1999; 545-61.

4. Prendiville W, Cullimore J, Norman S. Large loop excision of the transformation zone (LLETZ): a new method of management for women with cervical intraepithelial neoplasia. Br J Obstet Gynaecol 1989;96:1054-60.

5. Mathevet $P$, Dargent D, Roy M, Beau G. A randomized prospective study comparing three techniques of conization: cold knife, laser, and LEEP. Gynecol Oncol 1994;54:175-9.

6. Nuovo J, Melnikow J, Willan AR, Chan BK. Treatment outcomes for squamous intraepithelial lesions. Int J Gynaecol Obstet 2000;68:25-33.
7. Kristensen J, Langhoff-Roos J, Wittrup M, Bock JE. Cervical conization and preterm delivery/low birth weight. A systematic review ofthe literature. Acta Obstet Gynecol Scand 1993;72(8):640-4.

8. Arbyn M, Kyrgiou M, Simoens C, Raifu AO, Koliopoulos G, Martin-Hirsch P, Prendiville W, Paraskevaidis E. Perinatal mortality and other severe adverse pregnancy outcomes associated with treatment of cervical intraepithelial neoplasia: meta-analysis. BMJ 2008;337:a1284.

9. Kyrgiou M, Koliopoulos G, Martin-Hirsch P, Arbyn M, Prendiville W, Paraskevaidis E. Obstetric outcomes after conservative treatment for intraepithelial or early invasive cervical lesions: systematic review and meta-analysis. Lancet 2006;367:489-98.

10. Acharya G, Kjeldberg I, Hansen SM, Sørheim N, Jacobsen BK, Maltau JM. Pregnancy outcome after loop electrosurgical excision procedure for the management of cervical intraepithelial neoplasia. Arch Gynecol Obstet 2005;272(2):109-12.

11. Sadler L, Saftlas A, Wang W, Exeter M, Whittaker J, McCowan L. Treatment for cervical intraepithelial neoplasia and risk of preterm delivery. JAMA 2004;291(17):21006.

12. Samson SL, Bentley JR, Fahey TJ, McKay DJ, Gill $\mathrm{GH}$. The effect of loop electrosurgical excision procedure on future pregnancy outcome. Obstet Gynecol 2005;105(2):325-32.

13. Phillips B, Ball C, Sackett D, Badenoch D, Straus S, Hayness B, Dawes M. Oxford Centre for Evidence-based Medicine. Levels of Evidence and Grades of Recommendation. Hallado en: http://www.cebm.net/index. aspx?o=1025\#levels.

14. Svare JA, Anderson LF, Langhoff-Roos J, Jensen ET, Bruun B, Lind I, Madsen $\mathrm{H}$. The relationship between prior cervical conization, cervical microbial colonization and preterm premature rupture of the membranes. Eur $\mathrm{J}$ Obstet Gynecol Reprod Biol 1992;47:41-5. 